\title{
Regularity and Uniqueness of Solutions to Boundary Blow-up Problems for the Complex Monge-Ampère Operator \\ by
}

\section{Björn IVARSSON}

\author{
Presented by Józef SICIAK
}

Summary. We prove that plurisubharmonic solutions to certain boundary blow-up problems for the complex Monge-Ampère operator are Lipschitz continuous. We also prove that in certain cases these solutions are unique.

1. Introduction. In [3], Cheng and Yau studied the problem

$$
\left\{\begin{array}{l}
\operatorname{det}\left(\frac{\partial^{2} u}{\partial z_{j} \partial \bar{z}_{k}}(z)\right)=f(z) e^{K u(z)}, \quad z \in \Omega, \\
\lim _{z \rightarrow z_{0}} u(z)=\infty \text { for all } z_{0} \in \partial \Omega,
\end{array}\right.
$$

where $\Omega$ is a bounded strongly pseudoconvex domain in $\mathbb{C}^{n}$ with smooth boundary, $f$ is a smooth strictly positive function and $K>0$ a constant. They showed that there is a unique smooth plurisubharmonic solution to this problem. In this paper we study a similar problem, namely

$$
\left\{\begin{array}{l}
\operatorname{det}\left(\frac{\partial^{2} u}{\partial z_{j} \partial \bar{z}_{k}}(z)\right)=f(z, u(z)), \quad z \in \Omega, \\
\lim _{z \rightarrow z_{0}} u(z)=\infty \text { for all } z_{0} \in \partial \Omega,
\end{array}\right.
$$

where the right hand side is a function $f \in C^{\infty}(\bar{\Omega} \times \mathbb{R})$ which is strictly positive, increasing in the second variable and satisfies the following three conditions:

2000 Mathematics Subject Classification: 32W20, (32U10, 35B65, 35J60).

Key words and phrases: complex Monge-Ampère operator, interior regularity, plurisubharmonic function, strongly pseudoconvex domain. 
A: There exist functions $h \in C^{\infty}(\bar{\Omega})$ and $f_{1} \in C^{\infty}(\mathbb{R})$ and two strictly positive constants $c_{1}$ and $c_{2}$ such that

$$
\lim _{t \rightarrow \infty} \frac{f(z, t)}{f_{1}(t)}=h(z)
$$

uniformly in $\Omega$ and $c_{1} f_{1}(t) \leq f(z, t) \leq c_{2} f_{1}(t)$ for all $(z, t) \in \Omega \times \mathbb{R}$.

B: The function $f_{1}$ is strictly positive and increasing.

C: The function

$$
\Psi_{n}(a)=\int_{a}^{\infty}((n+1) F(y))^{-1 /(n+1)} d y
$$

exists for $a>0$, where $F^{\prime}(s)=f_{1}(s)$ and $F(0)=0$.

Certain aspects of this problem has been studied by the author and Matero in [7].

The following theorem proven by Caffarelli, Kohn, Nirenberg and Spruck in [2] will be useful.

THEOREM 1.1. Let $\Omega$ be a bounded, strongly pseudoconvex domain in $\mathbb{C}^{n}$ with smooth boundary. Let $f \in C^{\infty}(\bar{\Omega} \times \mathbb{R})$ be a strictly positive function which is increasing in the second variable. Let $\varphi \in C^{\infty}(\partial \Omega)$. Then the problem

$$
\left\{\begin{array}{l}
\operatorname{det}\left(\frac{\partial^{2} u}{\partial z_{j} \partial \bar{z}_{k}}\right)=f(z, u) \text { in } \Omega, \\
u=\varphi \text { on } \partial \Omega
\end{array}\right.
$$

has a unique strictly plurisubharmonic solution $u$. Moreover, $u \in C^{\infty}(\bar{\Omega})$.

This result is used to construct solutions to Problem (1). A sequence of plurisubharmonic functions $u_{N}$ which solve Problem (2) on certain pseudoconvex domains $\Omega_{N}$ is constructed. We construct upper and lower bounds for these solutions and since $\Omega=\bigcup_{N} \Omega_{N}$ we can conclude that the sequence $u_{N}$ converges to a solution for Problem (1) on $\Omega$. This is done in Section 2 . In Section 3 the regularity of the solution is studied in some special cases. There it is assumed that the right hand side $f$ depends only on $u$, that is, $f(z, u)=f(u)$, and also satisfies an extra condition. The extra assumption is used to get a priori estimates for the first derivatives of solutions, which lets us conclude that solutions to Problem (1) are Lipschitz under these assumptions. Finally, in Section 4 uniqueness of solutions is studied. Here the right hand side can depend on the $z$-variable but we need to make another extra assumption. This extra assumption together with estimates on the boundary behavior of the solution, which were proved in [7], lets us conclude that solutions to Problem (1) are unique. 
We will use the notation

$$
u_{j}=\frac{\partial u}{\partial z_{j}}, \quad u_{\bar{k}}=\frac{\partial u}{\partial \bar{z}_{k}}, \quad u_{j \bar{k}}=\frac{\partial^{2} u}{\partial z_{j} \partial \bar{z}_{k}} .
$$

2. Construction of solutions. In order to prove existence of a solution of the problem

$$
\left\{\begin{array}{c}
\operatorname{det}\left(u_{j \bar{k}}(z)\right)=f(z, u(z)), \quad z \in \Omega, \\
\lim _{z \rightarrow z_{0}} u(z)=\infty \text { for all } z_{0} \in \partial \Omega,
\end{array}\right.
$$

we shall begin by constructing approximate solutions. Let $\varrho: \Omega \rightarrow \mathbb{R}$ be a strictly negative plurisubharmonic function such that $\varrho \in C^{\infty}(\bar{\Omega})$ and $\lim _{z \rightarrow z_{0}} \varrho(z)=0$ for all $z_{0} \in \partial \Omega$. Take a strictly increasing convex function $g: \mathbb{R}^{-} \rightarrow \mathbb{R}$ such that $\lim _{x \rightarrow 0-} g(x)=\infty$. Put $\varphi(z)=g(\varrho(z))$. This is a plurisubharmonic function which satisfies $\lim _{z \rightarrow z_{0}} \varphi(z)=\infty$ for all $z_{0} \in \partial \Omega$. Let

$$
\left(\varrho^{j \bar{k}}\right)=\left(\varrho_{j \bar{k}}\right)^{-1}
$$

and

$$
\|d \varrho\|_{\varrho}^{2}=\varrho^{j \bar{k}} \varrho_{j} \varrho_{\bar{k}} .
$$

We see that

$$
\frac{\partial \varphi}{\partial \bar{z}_{k}}=\varrho_{\bar{k}} g^{\prime}(\varrho)
$$

and

$$
\varphi_{j \bar{k}}=\varrho_{j \bar{k}} g^{\prime}(\varrho)+\varrho_{j} \varrho_{\bar{k}} g^{\prime \prime}(\varrho) .
$$

Let $M_{j \bar{k}}$ be the minor

$$
\operatorname{det}\left(\begin{array}{cccccc}
\varrho_{1 \overline{1}} & \ldots & \varrho_{1 \overline{(k-1})} & \varrho_{1 \overline{(k+1)}} & \ldots & \varrho_{1 \bar{n}} \\
\vdots & \ddots & \vdots & \vdots & \ddots & \vdots \\
\varrho_{(j-1) \overline{1}} & \cdots & \varrho_{(j-1) \overline{(k-1)}} & \varrho_{(j-1) \overline{(k+1)}} & \cdots & \varrho_{(j-1) \bar{n}} \\
\varrho_{(j+1) \overline{1}} & \cdots & \varrho_{(j+1) \overline{(k-1)}} & \varrho_{(j+1) \overline{(k+1)}} & \cdots & \varrho_{(j+1) \bar{n}} \\
\vdots & \ddots & \vdots & \vdots & \ddots & \vdots \\
\varrho_{n \overline{1}} & \ldots & \varrho_{n \overline{(k-1)}} & \varrho_{n \overline{(k+1)}} & \ldots & \varrho_{n \bar{n}}
\end{array}\right) .
$$

We see that

$$
\begin{aligned}
\operatorname{det}\left(\varphi_{j \bar{k}}\right) & =\operatorname{det}\left(\varrho_{j \bar{k}} g^{\prime}(\varrho)+\varrho_{j} \varrho_{\bar{k}} g^{\prime \prime}(\varrho)\right) \\
& =g^{\prime}(\varrho)^{n} \operatorname{det}\left(\varrho_{j \bar{k}}\right)+g^{\prime \prime}(\varrho) g^{\prime}(\varrho)^{n-1} \sum_{j, k=1}^{n} M_{j k} \varrho_{j} \varrho_{\bar{k}} \\
& =\left(g^{\prime}(\varrho)^{n}+\|d \varrho\|_{\varrho}^{2} g^{\prime \prime}(\varrho) g^{\prime}(\varrho)^{n-1}\right) \operatorname{det}\left(\varrho_{j \bar{k}}\right)
\end{aligned}
$$


Since

$$
g^{\prime}(\varrho(z))=1 /\left(g^{-1}\right)^{\prime}(\varphi(z))
$$

and

$$
g^{\prime \prime}(\varrho(z))=-\left(g^{-1}\right)^{\prime \prime}(\varphi(z)) /\left(g^{-1}\right)^{\prime}(\varphi(z))^{3},
$$

this can be rewritten as

$$
\operatorname{det}\left(\varphi_{j \bar{k}}\right)=\frac{1}{\left(g^{-1}\right)^{\prime}(\varphi(z))^{n}} \operatorname{det}\left(\varrho_{j \bar{k}}\right)-\frac{\left(g^{-1}\right)^{\prime \prime}(\varphi(z))}{\left(g^{-1}\right)^{\prime}(\varphi(z))^{n+2}}\|d \varrho\|_{\varrho}^{2} \operatorname{det}\left(\varrho_{j \bar{k}}\right) .
$$

We shall show that we can choose $g$ so that

$$
\frac{1}{\left(g^{-1}\right)^{\prime}(\varphi(z))^{n}} \leq-\frac{\left(g^{-1}\right)^{\prime \prime}(\varphi(z))}{\left(g^{-1}\right)^{\prime}(\varphi(z))^{n+2}}\|d \varrho\|_{\varrho}^{2}
$$

near the boundary. This will show that the last term is the important term.

Hopf's lemma, sometimes also referred to as Zaremba's principle, implies that a plurisubharmonic function $u \in C^{1}(\bar{\Omega}) \cap C^{2}(\Omega)$ which satisfies $u(z)<$ $u\left(z_{0}\right)$ for all $z \in \Omega$ and a boundary point $z_{0}$ also satisfies $(\partial u / \partial \nu)\left(z_{0}\right)<0$ where $\nu$ denotes the inward-pointing normal to $\partial \Omega$. A proof of Hopf's lemma can be found in Taylor's book [9]. Since every boundary point is a global maximum for $\varrho$ and $\partial \Omega$ is compact we see that $\|d \varrho\|_{\varrho}^{2}>\varepsilon$, for some $\varepsilon>0$, near the boundary.

We are interested in solving

$$
\left\{\begin{array}{l}
\operatorname{det}\left(u_{j \bar{k}}(z)\right)=f(z, u(z)), \quad z \in \Omega, \\
\lim _{z \rightarrow z_{0}} u(z)=\infty \text { for all } z_{0} \in \partial \Omega
\end{array}\right.
$$

where $f$ is strictly positive, increasing in the second variable and satisfies conditions A, B and C. We deduce what $g$ should be by solving

$$
-\frac{\left(g^{-1}\right)^{\prime \prime}(x)}{\left(g^{-1}\right)^{\prime}(x)^{n+2}}=f_{1}(x) \text {. }
$$

Rewriting this we get

$$
\frac{d}{d x}\left(\frac{1}{(n+1)\left(g^{-1}\right)^{\prime}(x)^{n+1}}\right)=f_{1}(x) .
$$

Integrating we see that

$$
\frac{1}{\left(g^{-1}\right)^{\prime}(x)^{n+1}}=(n+1) F(x) .
$$

This implies that

$$
g^{-1}(x)=\int((n+1) F(x))^{-1 /(n+1)} d x .
$$

In particular, we can choose $g^{-1}(x)=-\Psi_{n}(x)$. Making this choice we get

$$
\left(g^{-1}\right)^{\prime}(x)=((n+1) F(x))^{-1 /(n+1)} .
$$


Let us now turn to the question if

$$
\frac{1}{\left(g^{-1}\right)^{\prime}(\varphi(z))^{n}} \leq-\frac{\left(g^{-1}\right)^{\prime \prime}(\varphi(z))}{\left(g^{-1}\right)^{\prime}(\varphi(z))^{n+2}}\|d \varrho\|_{\varrho}^{2}
$$

near the boundary. But this is the same as

$$
\varepsilon^{-1} \leq-\frac{\left(g^{-1}\right)^{\prime \prime}(x)}{\left(g^{-1}\right)^{\prime}(x)^{2}}=\frac{d}{d x}\left(\frac{1}{\left(g^{-1}\right)^{\prime}(x)}\right)
$$

as $x$ tends to $\infty$. Here $\varepsilon$ is the infimum of $\|d \varrho\|_{\varrho}^{2}$ in some neighborhood of the boundary. Assume that

$$
\frac{d}{d x}\left(\frac{1}{\left(g^{-1}\right)^{\prime}(x)}\right)=\frac{d}{d x}((n+1) F(x))^{1 /(n+1)}<\varepsilon^{-1}
$$

for large $x$. We get

$$
((n+1) F(x))^{1 /(n+1)}<\varepsilon^{-1} x+C
$$

for large $x$ but this contradicts the integrability of $((n+1) F(x))^{-1 /(n+1)}$. Hence

$$
\frac{d}{d x}\left(\frac{1}{\left(g^{-1}\right)^{\prime}(x)}\right) \geq \varepsilon^{-1}
$$

and we conclude that

$$
\frac{1}{\left(g^{-1}\right)^{\prime}(\varphi(z))^{n}} \leq-\frac{\left(g^{-1}\right)^{\prime \prime}(\varphi(z))}{\left(g^{-1}\right)^{\prime}(\varphi(z))^{n+2}}\|d \varrho\|_{\varrho}^{2}
$$

near the boundary.

Having this at our disposal we can construct plurisubharmonic functions which are approximate solutions to the problem we are interested in. Namely, given $f$ and $f_{1}$ use the method above to choose $g$. Take a plurisubharmonic function $\varrho$ which solves

$$
\left\{\begin{array}{l}
\operatorname{det}\left(\varrho_{j \bar{k}}(z)\right)=1, \quad z \in \Omega \\
\lim _{z \rightarrow z_{0}} \varrho(z)=0 \quad \text { for all } z_{0} \in \partial \Omega
\end{array}\right.
$$

By Theorem 1.1 we know that $\varrho \in C^{\infty}(\bar{\Omega})$. It is also strictly plurisubharmonic on $\bar{\Omega}$. Hence $\|d \varrho\|_{\varrho}^{2} \in C^{\infty}(\bar{\Omega})$. Put $\varphi=g \circ \varrho$. We see that $\lim _{z \rightarrow z_{0}} \varphi(z)=\infty$ for all $z_{0} \in \partial \Omega$ and

$$
\operatorname{det}\left(\varphi_{j \bar{k}}(z)\right)=\frac{1}{\left(g^{-1}\right)^{\prime}(\varphi)^{n}}-\frac{\left(g^{-1}\right)^{\prime \prime}(\varphi)}{\left(g^{-1}\right)^{\prime}(\varphi)^{n+2}}\|d \varrho\|_{\varrho}^{2}=\kappa(z) f_{1}(\varphi)
$$

where

$$
0<C \leq \kappa(z)=\|d \varrho\|_{\varrho}^{2}-\frac{\left(g^{-1}\right)^{\prime}(\varphi)^{2}}{\left(g^{-1}\right)^{\prime \prime}(\varphi)} \leq C^{\prime} .
$$

The existence of the upper bound $C^{\prime}$ is clear. The lower bound is a little trickier. At points near the boundary we know, by Hopf's lemma, that $\|d \varrho\|_{\varrho}^{2}>\varepsilon$. 
At points where $\|d \varrho\|_{\varrho}^{2} \leq \varepsilon$ (these points are not close to the boundary), we see that $\varphi$ is bounded and hence

$$
-\frac{\left(g^{-1}\right)^{\prime}(\varphi)^{2}}{\left(g^{-1}\right)^{\prime \prime}(\varphi)} \geq \varepsilon^{\prime}
$$

for some $\varepsilon^{\prime}>0$. We see that there is a lower bound $C$ so that $0<C<\kappa(z)$.

Now notice that $\varrho_{K}=K \varrho$ satisfies

$$
\left\{\begin{array}{l}
\operatorname{det}\left(\varrho_{K, j \bar{k}}(z)\right)=K^{n}, \quad z \in \Omega, \\
\lim _{z \rightarrow z_{0}} \varrho_{K}(z)=0 \text { for all } z_{0} \in \partial \Omega .
\end{array}\right.
$$

The function $\varphi_{K}=g \circ \varrho_{K}$ satisfies $g^{-1}\left(\varrho_{K}\right)=K g^{-1}(\varrho)$. Therefore

$$
\begin{aligned}
\operatorname{det}\left(\varphi_{K, j \bar{k}}(z)\right) & =\operatorname{det}\left(\varrho_{K, j \bar{k}}(z)\right)\left(\left\|d \varrho_{K}\right\|_{\varrho_{K}}^{2}-\frac{\left(g^{-1}\right)^{\prime}\left(\varphi_{K}\right)^{2}}{\left(g^{-1}\right)^{\prime \prime}\left(\varphi_{K}\right)}\right) f_{1}\left(\varphi_{K}\right) \\
& =K^{n}\left(K\|d \varrho\|_{\varrho}^{2}-\frac{\left(g^{-1}\right)^{\prime}\left(\varphi_{K}\right)^{2}}{\left(g^{-1}\right)^{\prime \prime}\left(\varphi_{K}\right)}\right) f_{1}\left(\varphi_{K}\right) \\
& =K^{n+1}\left(\|d \varrho\|_{\varrho_{K}}^{2}-\frac{\left(g^{-1}\right)^{\prime}(\varphi)^{2}}{\left(g^{-1}\right)^{\prime \prime}(\varphi)}\right) f_{1}\left(\varphi_{K}\right)=K^{n+1} \kappa(z) f_{1}\left(\varphi_{K}\right) .
\end{aligned}
$$

We see that by choosing $K$ and $\widetilde{K}$ suitably we have $\widetilde{K}^{n+1} \kappa \leq c_{1}$ and $c_{2} \leq$ $K^{n+1} \kappa$. Let $\Omega_{N}=\left\{z \in \Omega ; \varphi_{K}(z)<N\right\}$ and $u_{N}$ be the solution of

$$
\left\{\begin{array}{l}
\operatorname{det}\left(u_{N, j \bar{k}}(z)\right)=f\left(z, u_{N}(z)\right), \quad z \in \Omega_{N}, \\
\lim _{z \rightarrow z_{0}} u_{N}(z)=N \quad \text { for all } z_{0} \in \partial \Omega_{N} .
\end{array}\right.
$$

which exists by Theorem 1.1. By Lemma 2.2 in [6] we get $\varphi_{K} \leq u_{N} \leq$ $u_{N+1} \leq \varphi_{\widetilde{K}}$ on $\Omega_{N}$. Define $u(z)=\lim _{N \rightarrow \infty} u_{N}(z)$. We now investigate the regularity of $u$.

3. A priori estimate of first derivatives of solutions. In this section we assume that $f(z, u)=f(u)$ is a function satisfying $\mathbf{B}, \mathbf{C}$ and the technical condition

$$
\frac{n-1}{n+1} \leq \frac{F(x) f^{\prime}(x)}{f(x)^{2}}
$$

We shall estimate the norm of the gradient of $u_{N}$ on compact subsets of $\Omega$. We do this by studying the functions $v_{N}=\left|\nabla u_{N}\right|^{2}\left(g^{-1}\right)^{\prime}\left(u_{N}\right)^{2}$. Notice that $\left|\nabla \varrho_{K}\right|^{2}=|\nabla \varphi|^{2}\left(g^{-1}\right)^{\prime}(\varphi)^{2} \leq C$ and that $v_{N}=\left|\nabla u_{N}\right|^{2}\left(g^{-1}\right)^{\prime}\left(u_{N}\right)^{2} \leq$ $|\nabla \varphi|^{2}\left(g^{-1}\right)^{\prime}(\varphi)^{2}$ on $\partial \Omega_{N}$ since $u_{N}=\varphi$ on $\partial \Omega_{N}$ and $\varphi \leq u_{N}$ in $\Omega_{N}$. We claim that $\sup \left(v_{N}(z) ; z \in \Omega_{N}\right) \leq \sup \left(v_{N}(z) ; z \in \partial \Omega_{N}\right) \leq C$. We shall show that $v_{N}$ does not have any interior maximum in $\Omega_{N}$ to establish the claim. This calculation was inspired by Bo Guan's work on the regularity of the pluricomplex Green function [4], [5]. Readers interested in the regularity of the pluricomplex Green function should also consult Błocki's paper [1]. 
Assume that a local maximum for $v_{N}$ is attained at $p \in \Omega_{N}$. We know that $\nabla v_{N}(p)=0$. Choose coordinates near $p$ so that $u_{N, j k}(p)=u_{N, \overline{j k}}(p)=0$ and $u_{N, j \bar{k}}(p)=0$ if $j \neq k$. It is known that such coordinates can be found if $\nabla u_{N}(p) \neq 0$, which is the case at a maximum point of $v_{N}$. A proof can be extracted from the calculation on page 130 of [8]. Remember that

$$
v_{N}=\sum_{l=1}^{n} u_{N, l} u_{N, \bar{l}}\left(g^{-1}\right)^{\prime}\left(u_{N}\right)^{2}
$$

and hence

$$
\begin{aligned}
v_{N, j}= & \sum_{l=1}^{n}\left(u_{N, l} u_{N, j \bar{l}}\left(g^{-1}\right)^{\prime}\left(u_{N}\right)^{2}\right. \\
& \left.+u_{N, j l} u_{N, \bar{l}}\left(g^{-1}\right)^{\prime}\left(u_{N}\right)^{2}+2\left(g^{-1}\right)^{\prime}\left(u_{N}\right)\left(g^{-1}\right)^{\prime \prime}\left(u_{N}\right) u_{N, l} u_{N, \bar{l}} u_{N, j}\right) .
\end{aligned}
$$

Evaluating this at $p$ yields

$$
\begin{aligned}
v_{N, j} & =\sum_{l=1}^{n}\left(u_{N, l} u_{N, j \bar{l}}\left(g^{-1}\right)^{\prime}\left(u_{N}\right)^{2}+2\left(g^{-1}\right)^{\prime}\left(u_{N}\right)\left(g^{-1}\right)^{\prime \prime}\left(u_{N}\right) u_{N, l} u_{N, \bar{l}} u_{N, j}\right) \\
& =u_{N, j} u_{N, j \bar{j}}\left(g^{-1}\right)^{\prime}\left(u_{N}\right)^{2}+\sum_{l=1}^{n} 2\left(g^{-1}\right)^{\prime}\left(u_{N}\right)\left(g^{-1}\right)^{\prime \prime}\left(u_{N}\right) u_{N, l} u_{N, \bar{l}} u_{N, j} \\
& =u_{N, j}\left(g^{-1}\right)^{\prime}\left(u_{N}\right)^{2}\left(u_{N, j \bar{j}}+2 \frac{\left(g^{-1}\right)^{\prime \prime}\left(u_{N}\right)}{\left(g^{-1}\right)^{\prime}\left(u_{N}\right)}\left|\nabla u_{N}\right|^{2}\right)=0 .
\end{aligned}
$$

At the relevant local maximum point we have $\left|\nabla u_{N}\right|>0$ and therefore

$$
\prod_{j=1}^{n}\left(u_{N, j \bar{j}}+2 \frac{\left(g^{-1}\right)^{\prime \prime}\left(u_{N}\right)}{\left(g^{-1}\right)^{\prime}\left(u_{N}\right)}\left|\nabla u_{N}\right|^{2}\right)=0 .
$$

Thus we have

$$
\left|\nabla u_{N}\right|^{2}=-\frac{\left(g^{-1}\right)^{\prime}\left(u_{N}\right)}{2\left(g^{-1}\right)^{\prime \prime}\left(u_{N}\right)} u_{N, j \bar{j}}
$$

for some $j$. We see that

$$
\left|\nabla u_{N}\right|^{2} \leq-\frac{\left(g^{-1}\right)^{\prime}\left(u_{N}\right)}{2\left(g^{-1}\right)^{\prime \prime}\left(u_{N}\right)} \sum_{j=1}^{n} u_{N, j \bar{j}}
$$

with equality if and only if $u_{N, j \bar{j}}=0$ for all but one $j$. Hence

$$
\left|\nabla u_{N}\right|^{2}<-\frac{\left(g^{-1}\right)^{\prime}\left(u_{N}\right)}{2\left(g^{-1}\right)^{\prime \prime}\left(u_{N}\right)} \sum_{j=1}^{n} u_{N, j \bar{j}}
$$

because otherwise $\operatorname{det}\left(u_{N, j \bar{k}}\right)=0$. Remembering that

$$
\left(g^{-1}\right)^{\prime}(x)=((n+1) F(x))^{-1 /(n+1)}
$$


we get

$$
\left|\nabla u_{N}\right|^{2}<\frac{(n+1) F\left(u_{N}\right)}{2 f\left(u_{N}\right)} \sum_{j=1}^{n} u_{N, j \bar{j}}
$$

So far we have only used the fact that $p$ is a critical point. Now we shall use the fact that it is a local maximum point. We have $\log \operatorname{det}\left(u_{N, j \bar{k}}\right)=$ $\log f(u)$. Differentiating we see that

$$
\frac{\partial}{\partial z_{j}} \log \operatorname{det}\left(u_{N, k \bar{l}}\right)=\sum_{k, l=1}^{n} \frac{M_{k \bar{l}}}{\operatorname{det}\left(u_{N, k \bar{l}}\right)} u_{N, k \bar{l} j}=\sum_{k, l=1}^{n} u_{N}^{k \bar{l}} u_{N, k \bar{l} j}=\sum_{l=1}^{n} u_{N}^{l \bar{l}} u_{N, l \bar{l} j}
$$

and hence we get the relation

$$
\sum_{l=1}^{n} u_{N}^{l \bar{l}} u_{N, l \bar{l} j}=\frac{f^{\prime}\left(u_{N}\right)}{f\left(u_{N}\right)} u_{N, j}
$$

We also have

$$
\sum_{l=1}^{n} u_{N}^{l \bar{l}} u_{N, l \overline{l j}}=\frac{f^{\prime}\left(u_{N}\right)}{f\left(u_{N}\right)} u_{N, \bar{j}}
$$

If we differentiate $v_{N}$ twice we get

$$
\begin{aligned}
v_{N, j \bar{k}}= & \left(g^{-1}\right)^{\prime}\left(u_{N}\right)^{2} \sum_{l=1}^{n}\left(u_{N, l j \bar{k}} u_{N, \bar{l}}+u_{N, l \bar{k}} u_{N, j \bar{l}}+u_{N, l} u_{N, j \overline{l k}}\right) \\
& +2\left(g^{-1}\right)^{\prime}\left(u_{N}\right)\left(g^{-1}\right)^{\prime \prime}\left(u_{N}\right) u_{N, \bar{k}} \sum_{l=1}^{n} u_{N, l} u_{N, j \bar{l}} \\
& +2\left(g^{-1}\right)^{\prime}\left(u_{N}\right)\left(g^{-1}\right)^{\prime \prime}\left(u_{N}\right) u_{N, j} \sum_{l=1}^{n} u_{N, l \bar{k}} u_{N, \bar{l}} \\
& +2\left(g^{-1}\right)^{\prime}\left(u_{N}\right)\left(g^{-1}\right)^{\prime \prime}\left(u_{N}\right) u_{N, j \bar{k}} \sum_{l=1}^{n} u_{N, l} u_{N, \bar{l}} \\
& +\left(2\left(g^{-1}\right)^{\prime}\left(u_{N}\right)\left(g^{-1}\right)^{\prime \prime \prime}\left(u_{N}\right)+2\left(g^{-1}\right)^{\prime \prime}\left(u_{N}\right)^{2}\right) u_{N, j} u_{N, \bar{k}} \sum_{l=1}^{n} u_{N, l} u_{N, \bar{l}} .
\end{aligned}
$$

Here we have used the fact that the Hessian of $u_{N}$ is diagonal to simplify the expression. Since $p$ is assumed to be a local maximum point we know that

$$
\sum_{j, k=1}^{n} u_{N}^{j \bar{k}} v_{N, j \bar{k}} \leq 0
$$


Therefore

$$
\begin{aligned}
& \sum_{j, k=1}^{n} u_{N}^{j \bar{k}} v_{N, j \bar{k}}=\sum_{j=1}^{n} u_{N}^{j \bar{j}} v_{N, j \bar{j}} \\
& =\left(g^{-1}\right)^{\prime}\left(u_{N}\right)^{2}\left(\sum_{j, l=1}^{n}\left(u_{N}^{j \bar{j}} u_{N, l j \bar{j}} u_{N, \bar{l}}+u_{N}^{j \bar{j}} u_{N, l} u_{N, j \overline{l j}}\right)+\sum_{j=1}^{n} u_{N, j \bar{j}}\right) \\
& \quad+(4+2 n)\left(g^{-1}\right)^{\prime}\left(u_{N}\right)\left(g^{-1}\right)^{\prime \prime}\left(u_{N}\right) \sum_{j=1}^{n} u_{N, \bar{j}} u_{N, j} \\
& \quad+\left(2\left(g^{-1}\right)^{\prime}\left(u_{N}\right)\left(g^{-1}\right)^{\prime \prime \prime}\left(u_{N}\right)+2\left(g^{-1}\right)^{\prime \prime}\left(u_{N}\right)^{2}\right) \sum_{j, l=1}^{n} u_{N}^{j \bar{j}} u_{N, j} u_{N, \bar{j}} u_{N, l} u_{N, \bar{l}} \\
& =2\left(g^{-1}\right)^{\prime}\left(u_{N}\right)^{2} \frac{f^{\prime}\left(u_{N}\right)}{f\left(u_{N}\right)}\left|\nabla u_{N}\right|^{2}+\left(g^{-1}\right)^{\prime}\left(u_{N}\right)^{2} \sum_{j=1}^{n} u_{N, j \bar{j}} \\
& \quad+(4+2 n)\left(g^{-1}\right)^{\prime}\left(u_{N}\right)\left(g^{-1}\right)^{\prime \prime}\left(u_{N}\right)\left|\nabla u_{N}\right|^{2} \\
& \quad+\left(2\left(g^{-1}\right)^{\prime}\left(u_{N}\right)\left(g^{-1}\right)^{\prime \prime \prime}\left(u_{N}\right)+2\left(g^{-1}\right)^{\prime \prime}\left(u_{N}\right)^{2}\right)\left|\nabla u_{N}\right|^{2} \sum_{j=1}^{n} u_{N}^{j \bar{j}} u_{N, j} u_{N, \bar{j}} \leq 0
\end{aligned}
$$

at $p$. We need to analyze $\sum_{j=1}^{n} u_{N}^{j \bar{j}} u_{N, j} u_{N, \bar{j}}$. At $p$ we have

$$
u_{N, j \bar{j}}=-2 \frac{\left(g^{-1}\right)^{\prime \prime}\left(u_{N}\right)}{\left(g^{-1}\right)^{\prime}\left(u_{N}\right)}\left|\nabla u_{N}\right|^{2}
$$

if $u_{N, j} \neq 0$. Therefore

$$
\sum_{j=1}^{n} u_{N}^{j \bar{j}} u_{N, j} u_{N, \bar{j}}=\sum_{j=1}^{n} \frac{u_{N, j} u_{N, \bar{j}}}{u_{N, j \bar{j}}}=-\frac{\left(g^{-1}\right)^{\prime}\left(u_{N}\right)}{2\left(g^{-1}\right)^{\prime \prime}\left(u_{N}\right)} .
$$

Using this gives the inequality

$$
\sum_{j=1}^{n} u_{N, j \bar{j}} \leq\left|\nabla u_{N}\right|^{2}\left(\frac{\left(g^{-1}\right)^{\prime \prime \prime}\left(u_{N}\right)}{\left(g^{-1}\right)^{\prime \prime}\left(u_{N}\right)}-\frac{2 f^{\prime}\left(u_{N}\right)}{f\left(u_{N}\right)}-\frac{(3+2 n)\left(g^{-1}\right)^{\prime \prime}\left(u_{N}\right)}{\left(g^{-1}\right)^{\prime}\left(u_{N}\right)}\right) .
$$

We have

$$
\begin{aligned}
\left(g^{-1}\right)^{\prime}(x)= & ((n+1) F(x))^{-1 /(n+1)} \\
\left(g^{-1}\right)^{\prime \prime}(x)= & -f(x)((n+1) F(x))^{-1-1 /(n+1)} \\
\left(g^{-1}\right)^{\prime \prime \prime}(x)= & -f^{\prime}(x)((n+1) F(x))^{-1-1 /(n+1)} \\
& +(n+2) f(x)^{2}((n+1) F(x))^{-2-1 /(n+1)}
\end{aligned}
$$


Hence

$$
\begin{aligned}
& \frac{\left(g^{-1}\right)^{\prime \prime \prime}\left(u_{N}\right)}{\left(g^{-1}\right)^{\prime \prime}\left(u_{N}\right)}-\frac{2 f^{\prime}\left(u_{N}\right)}{f\left(u_{N}\right)}-\frac{(3+2 n)\left(g^{-1}\right)^{\prime \prime}\left(u_{N}\right)}{\left(g^{-1}\right)^{\prime}\left(u_{N}\right)} \\
& \quad=\frac{\left(g^{-1}\right)^{\prime \prime \prime}\left(u_{N}\right)}{\left(g^{-1}\right)^{\prime \prime}\left(u_{N}\right)}-\frac{2 f^{\prime}\left(u_{N}\right)}{f\left(u_{N}\right)}+\frac{(3+2 n) f\left(u_{N}\right)}{(n+1) F\left(u_{N}\right)}=-\frac{f^{\prime}\left(u_{N}\right)}{f\left(u_{N}\right)}+\frac{f\left(u_{N}\right)}{F\left(u_{N}\right)} .
\end{aligned}
$$

Combining the two inequalities

$$
\left|\nabla u_{N}\right|^{2}<\frac{(n+1) F\left(u_{N}\right)}{2 f\left(u_{N}\right)} \sum_{j=1}^{n} u_{N, j \bar{j}}
$$

and

$$
\sum_{j=1}^{n} u_{N, j \bar{j}} \leq\left|\nabla u_{N}\right|^{2}\left(\frac{f\left(u_{N}\right)}{F\left(u_{N}\right)}-\frac{f^{\prime}\left(u_{N}\right)}{f\left(u_{N}\right)}\right)
$$

yields

$$
\left|\nabla u_{N}\right|^{2}<\frac{n+1}{2} \frac{F\left(u_{N}\right)}{f\left(u_{N}\right)} \sum_{j=1}^{n} u_{N, j \bar{j}} \leq \frac{n+1}{2}\left(1-\frac{F\left(u_{N}\right) f^{\prime}\left(u_{N}\right)}{f\left(u_{N}\right)^{2}}\right)\left|\nabla u_{N}\right|^{2},
$$

which gives a contradiction if

$$
\frac{n+1}{2}\left(1-\frac{F\left(u_{N}\right) f^{\prime}\left(u_{N}\right)}{f\left(u_{N}\right)^{2}}\right) \leq 1 .
$$

We see that, on the assumption

$$
\frac{n-1}{n+1} \leq \frac{F\left(u_{N}\right) f^{\prime}\left(u_{N}\right)}{f\left(u_{N}\right)^{2}}
$$

the function $\left|\nabla u_{N}\right|^{2}\left(g^{-1}\right)^{\prime}\left(u_{N}\right)^{2}$ attains its maximum on the boundary and hence we have

$$
\left|\nabla u_{N}\right|^{2}\left(g^{-1}\right)^{\prime}\left(u_{N}\right)^{2} \leq C
$$

on $\Omega_{N}$. Since any compact set $K \subseteq \Omega$ is contained in $\Omega_{N}$ for sufficiently large $N$ we have proven that

$$
\sup \left(\left|\nabla u_{N}(z)\right|^{2}\left(g^{-1}\right)^{\prime}\left(u_{N}(z)\right) ; z \in K\right)<C
$$

for all $N$ large enough. Hence

$$
\left|\nabla u_{N}(z)\right|^{2} \leq C g^{\prime}\left(u_{N}(z)\right)^{2}
$$

in $K$ and since $u_{N}(z) \leq \varphi(z) \leq C$ in $K$ we see that $\left\|u_{N}\right\|_{C^{1}(K)} \leq C$. Since the sequence of $u_{N}$ 's converges uniformly on compacts we can conclude that $u$ is Lipschitz. We state this in a theorem.

TheOREM 3.1. Let $\Omega$ be a bounded strongly pseudoconvex domain in $\mathbb{C}^{n}$ with smooth boundary. Suppose that $f$ satisfies $\mathbf{B}, \mathbf{C}$ and

$$
\frac{n-1}{n+1} \leq \frac{f^{\prime}(x) F(x)}{f(x)^{2}}
$$


Then the problem

$$
\left\{\begin{array}{l}
\left(d d^{c} u\right)^{n}=f(u(z)), \quad z \in \Omega \\
\lim _{z \rightarrow z_{0}} u(z)=\infty \quad \text { for all } z_{0} \in \partial \Omega
\end{array}\right.
$$

has a solution $u$ that is Lipschitz.

REMARK 3.2. Note that $f(u)=e^{K u}, K>0$, and $f(u)=u^{\gamma}$ (suitably modified for $u<1)$ where $\gamma \geq(n-1) / 2$, satisfies all the conditions in the theorem.

4. Uniqueness. We shall now establish a uniqueness result. Uniqueness for boundary blow-up problems is not as straightforward as for the Dirichlet problem. This is because the comparison principles in [6] and [7] are not formulated with the situation in mind where both plurisubharmonic functions tend to $\infty$ as we approach the boundary.

We need the following definition and theorem from [7].

Definition 4.1. Assume that $\Omega=\left\{z \in \mathbb{C}^{n} ; \varrho(z)<0\right\}$ where $\varrho \in$ $C^{\infty}(\bar{\Omega})$. For $z_{0} \in \partial \Omega$ suppose that $\left|\nabla \varrho\left(z_{0}\right)\right|=1$. Let $\Pi\left(z_{0}\right)$ be the product of the eigenvalues of the form

$$
\sum_{j, k=1}^{n} \frac{\partial^{2} \varrho}{\partial z_{j} \partial \bar{z}_{k}}\left(z_{0}\right) d z_{j} \wedge d \bar{z}_{k}
$$

restricted to the vector space $\left\{w \in \mathbb{C}^{n} ; \sum_{j=1}^{n} \frac{\partial \varrho}{\partial z_{j}}\left(z_{0}\right) w_{j}=0\right\}$.

THEOREM 4.2. Let $\Omega$ be a bounded, strongly pseudoconvex domain in $\mathbb{C}^{n}$ with smooth boundary. Let $f \in C^{\infty}(\bar{\Omega} \times \mathbb{R})$ be a strictly positive function which is increasing in the second variable and satisfies assumptions $\mathbf{A}, \mathbf{B}$ and $\mathbf{C}$. For boundary points $z_{0} \in \partial \Omega$ let $\Pi\left(z_{0}\right)$ be the number described in Definition 4.1. Then any solution u to Problem (1) satisfies

$$
\lim _{z \rightarrow z_{0}} \frac{\Psi_{n}(u(z))}{d_{\Omega}(z)}=4^{1 /(n+1)} h\left(z_{0}\right)^{1 /(n+1)} \Pi\left(z_{0}\right)^{-1 /(n+1)}
$$

for any $z_{0} \in \partial \Omega$.

We can now prove the following proposition.

Proposition 4.3. Let $\Omega$ be a bounded strongly pseudoconvex domain with smooth boundary and assume that $f \in C^{\infty}(\bar{\Omega} \times \mathbb{R})$ is a strictly positive function, increasing in the second variable and satisfying $\mathbf{A}, \mathbf{B}$ and $\mathbf{C}$. Assume also that

$$
\Psi_{n}(t) / \Psi_{n}^{\prime}(t)
$$

is bounded for large $t$. If $u$ and $v$ are plurisubharmonic solutions of Problem (1) then $u \equiv v$. 
REMARK 4.4. The assumption that $\Psi_{n}(t) / \Psi_{n}^{\prime}(t)$ is bounded for large $t$ is fulfilled when $f_{1}$ has exponential growth but not when it has only polynomial growth.

Proof of Proposition 4.3. Assume that we have two distinct plurisubharmonic solutions $u$ and $v$ of Problem (1). Assume for the moment that we know that $\lim _{z \rightarrow z_{0}}(u(z)-v(z))=0$ for all $z_{0} \in \partial \Omega$. We shall return to this claim later to finish the proof. Assume that $\sup (u(z)-v(z) ; z \in \Omega)=$ $K>0$. Then there is a $p \in \Omega$ such that $u(p)-v(p)=K$. At $p$ we have $\operatorname{det}\left(u_{j \bar{k}}(p)\right) \leq \operatorname{det}\left(v_{j \bar{k}}(p)\right)$. However, since $u(p)>v(p)$ we see that

$$
\operatorname{det}\left(u_{j \bar{k}}(p)\right)=f(p, u(p))>f(p, v(p))=\operatorname{det}\left(v_{j \bar{k}}(p)\right),
$$

which is a contradiction. Hence $u(z)-v(z) \leq 0$ in $\Omega$. Arguing in the same way we also see that $v(z)-u(z) \leq 0$ in $\Omega$. This proves uniqueness.

It remains to prove our claim that $\lim _{z \rightarrow z_{0}}(u(z)-v(z))=0$. We know that for all $z_{0} \in \partial \Omega$ we have

$$
\lim _{z \rightarrow z_{0}} \frac{\Psi_{n}(u(z))}{d_{\Omega}(z)}=\lim _{z \rightarrow z_{0}} \frac{\Psi_{n}(v(z))}{d_{\Omega}(z)}=C\left(z_{0}\right)
$$

where $C\left(z_{0}\right)$ is the constant given in Theorem 4.2. Given $\varepsilon>0$, for $z$ close to $z_{0}$ we have

$$
\left(C\left(z_{0}\right)-\varepsilon\right) d_{\Omega}(z) \leq \Psi_{n}(u(z)) \leq\left(C\left(z_{0}\right)+\varepsilon\right) d_{\Omega}(z)
$$

and

$$
\left(C\left(z_{0}\right)-\varepsilon\right) d_{\Omega}(z) \leq \Psi_{n}(v(z)) \leq\left(C\left(z_{0}\right)+\varepsilon\right) d_{\Omega}(z) .
$$

This gives

$$
\Psi_{n}^{-1}\left(\left(C\left(z_{0}\right)+\varepsilon\right) d_{\Omega}(z)\right) \leq u(z) \leq \Psi_{n}^{-1}\left(\left(C\left(z_{0}\right)-\varepsilon\right) d_{\Omega}(z)\right)
$$

and

$$
-\Psi_{n}^{-1}\left(\left(C\left(z_{0}\right)-\varepsilon\right) d_{\Omega}(z)\right) \leq-v(z) \leq-\Psi_{n}^{-1}\left(\left(C\left(z_{0}\right)+\varepsilon\right) d_{\Omega}(z)\right) .
$$

We get

$$
\begin{aligned}
u(z)-v(z) & \leq \Psi_{n}^{-1}\left(\left(C\left(z_{0}\right)-\varepsilon\right) d_{\Omega}(z)\right)-\Psi_{n}^{-1}\left(\left(C\left(z_{0}\right)+\varepsilon\right) d_{\Omega}(z)\right) \\
& =-2 \varepsilon d_{\Omega}(z)\left(\Psi_{n}^{-1}\right)^{\prime}(\eta(z))
\end{aligned}
$$

for some $\eta(z) \in\left[\left(C\left(z_{0}\right)-\varepsilon\right) d_{\Omega}(z),\left(C\left(z_{0}\right)+\varepsilon\right) d_{\Omega}(z)\right]$ by the mean-value theorem. Hence

$$
\begin{aligned}
u(z)-v(z) & \leq-2 \varepsilon d_{\Omega}(z)\left(\Psi_{n}^{-1}\right)^{\prime}(\eta(z))=-\frac{2 \varepsilon d_{\Omega}(z)}{\eta(z)} \eta(z) \frac{1}{\Psi_{n}^{\prime}\left(\Psi_{n}^{-1}(\eta(z))\right)} \\
& =-\frac{2 \varepsilon d_{\Omega}(z)}{\eta(z)} \frac{\Psi_{n}\left(\Psi_{n}^{-1}(\eta(z))\right)}{\Psi_{n}^{\prime}\left(\Psi_{n}^{-1}(\eta(z))\right)} \leq-\frac{2 \varepsilon}{C\left(z_{0}\right)-\varepsilon} \frac{\Psi_{n}\left(\Psi_{n}^{-1}(\eta(z))\right)}{\Psi_{n}^{\prime}\left(\Psi_{n}^{-1}(\eta(z))\right)} .
\end{aligned}
$$

The assumption that $\Psi_{n}(t) / \Psi_{n}^{\prime}(t)$ is bounded for large $t$ lets us conclude that, since $\varepsilon$ is arbitrary, $\lim _{z \rightarrow z_{0}}(u(z)-v(z))=0$. 
Acknowledgements. I would like to thank Tobias Ekholm, Christer Kiselman and Jerk Matero for their help during the writing of this paper. Also I would like to thank Frank Kutzschebauch for making it possible for me to visit the University of Bern.

\section{References}

[1] Z. Błocki, The $C^{1,1}$ regularity of the pluricomplex Green function, Michigan Math. J. 47 (2000), 211-215.

[2] L. Caffarelli, J. J. Kohn, L. Nirenberg and J. Spruck, The Dirichlet problem for nonlinear second order elliptic equations, II. Complex Monge-Ampère, and uniformly elliptic, equations, Comm. Pure Appl. Math. 38 (1985), 209-252.

[3] S.-Y. Cheng and S.-T. Yau, On the existence of a complete Kähler metric on noncompact complex manifolds and the regularity of Fefferman's equation, Comm. Pure Appl. Math. 33 (1980), 507-544.

[4] B. Guan, The Dirichlet problem for complex Monge-Ampère equations and regularity of the pluri-complex Green function, Comm. Anal. Geom. 6 (1998), 687-703.

[5] -, A correction to "The Dirichlet problem for complex Monge-Ampère equations and regularity of the pluri-complex Green function", ibid. 8 (2000), 213-218.

[6] B. Ivarsson, Interior regularity of solutions to a complex Monge-Ampère equation, Ark. Mat. 40 (2002), 275-300.

[7] B. Ivarsson and J. Matero, The blow-up rate of solutions to boundary blow-up problems for the complex Monge-Ampère operator, submitted, 2005, 18 pp.

[8] S. Krantz, Function Theory of Several Complex Variables, Wiley, 1982.

[9] M. Taylor, Partial Differential Equations: Basic Theory, Springer, 1996.

\section{Björn Ivarsson}

Mathematisches Institute

Universität Bern

Sidlerstrasse 5

CH-3012 Bern, Switzerland

E-mail: bjoern.ivarsson@math.unibe.ch

Received November 30, 2005;

received in final form June 1, 2006 